\title{
APPROXIMATION BY ENTIRE FUNCTIONS BELONGING TO THE LAGUERRE-PÓLYA CLASS
}

\author{
D. Dryanov and Q. I. Rahman \\ Dedicated to Richard Askey on the occasion of his 65th birthday
}

\begin{abstract}
For each given $p \geq 1$, we minimize the integral $\int_{-1}^{1}|1-f(x)|^{p} d x$ over all entire functions $f$ belonging to the Laguerre-Pólya class having $x=-1$ and $x=1$ as consecutive zeros. The extremal function turns out to be of the form $c\left(1-x^{2}\right)$ where $c$ depends on $p$. The case $p=1$ of the problem was considered earlier by J. G. Clunie.
\end{abstract}

\section{Introduction}

During the course of a lecture on convergence properties of polynomials with only real zeros, given at the Université de Montréal a few years ago, J. G. Clunie mentioned the following proposition as a lemma crucial for the proof of the main result which gave a necessary and sufficient condition for an entire function to belong to the well-known Laguerre-Pólya class.

Proposition 1. Let $\mathcal{P}_{\mathrm{R}}$ denote the class of all polynomials with only real zeros, having $x=-1$ and $x=1$ as consecutive zeros. If $f \in \mathcal{P}_{\mathrm{R}}$, then

$$
\int_{-1}^{1}|1-f(x)| d x>\frac{1}{10}
$$

For reasons explained in [2, p. 110], the manuscript on which the lecture was based has never been submitted for publication. This is why this inequality did not get the attention it might have otherwise drawn. For Clunie, it was enough to know that for $f$ belonging to $\mathcal{P}_{\mathrm{R}}$, the integral $\int_{-1}^{1}|1-f(x)| d x$ was bounded below by a positive constant not depending on $f$; but once such an inequality is discovered, it becomes natural to look for its sharp version. While considering another problem for polynomials in $\mathcal{P}_{\mathrm{R}}$, we needed to know (see the proof of Theorem 2 of the present paper) the infimum of $\int_{-1}^{1}|1-f(x)|^{2} d x$ over all polynomials belonging to $\mathcal{P}_{\mathrm{R}}$. We then found it natural to consider the problem of minimizing $\int_{-1}^{1}|1-f(x)|^{p} d x$ where $p \in(0, \infty)$ and $f \in \mathcal{P}_{\mathrm{R}}$.

If $f$ is negative on $(-1,1)$, then $\int_{-1}^{1}|1-(-f(x))|^{p} d x<\int_{-1}^{1}|1-f(x)|^{p} d x$ for all $p>0$, so if $\mathcal{P}_{\mathrm{R}^{+}}$consists of those functions in $\mathcal{P}_{\mathrm{R}}$ which are positive on $(-1,1)$, then

$$
\inf _{f \in \mathcal{P}_{\mathrm{R}}} \int_{-1}^{1}|1-f(x)|^{p} d x=\inf _{f \in \mathcal{P}_{\mathrm{R}^{+}}} \int_{-1}^{1}|1-f(x)|^{p} d x .
$$

Received March 10, 1998.

1991 Mathematics Subject Classification: 30D99, 41A10, 41A17, 41A44.

Key words and phrases: Polynomials with only real zeros, Laguerre-Pólya class, entire functions. 
At this stage, it seems desirable to mention that an entire function is said to belong to the Laguerre-Pólya class, $\mathfrak{L}-\mathfrak{P}$ for short, if it is the local uniform limit in $\mathbb{C}$ of a sequence of polynomials with only real zeros (see [1] and some of the literature cited therein for additional facts about $\mathfrak{L}-\mathfrak{P})$. Let us denote by $(\mathfrak{L}-\mathfrak{P})_{1}$ the set of all functions in $\mathfrak{L}-\mathfrak{P}$ which have $x=-1$ and $x=1$ as consecutive zeros and are positive on $(-1,1)$. A function $f$ in $(\mathfrak{L}-\mathfrak{P})_{1}$ can be written as $f(z)=\left(1-z^{2}\right) \psi(z)$ where

$$
\psi(z)=c e^{-a z^{2}+b z} \prod_{k=1}^{\infty}\left(1-t_{k} z\right) e^{t_{k} z}, \quad c>0, a \geq 0, b \in \mathbb{R},
$$

and $-1 \leq t_{k} \leq 1$ for $k=1,2,3, \ldots$ such that $\sum_{k=1}^{\infty} t_{k}^{2}<\infty$. Note that $f \in(\mathfrak{L}-\mathfrak{P})_{1}$ if and only if it is the local uniform limit in $\mathbb{C}$ of a sequence of polynomials in $\mathcal{P}_{\mathrm{R}^{+}}$.

\section{Statement of results}

We prove:

Theorem 1. For each $p \in[1, \infty)$, there exists a unique constant $c_{p}>0$ such that for every $f \in(\mathfrak{L}-\mathfrak{P})_{1}$, different from $c_{p}\left(1-x^{2}\right)$, we have

$$
\int_{-1}^{1}|1-f(x)|^{p} d x>\int_{-1}^{1}\left|1-c_{p}\left(1-x^{2}\right)\right|^{p} d x .
$$

Remark 1 . For each $p$, the quantity on the right-hand side is a positive number which is independent of $f$. It is easily seen that $c_{1}=1 /\left(1-4 \sin ^{2} \pi / 18\right)$ and

$$
\int_{-1}^{1}\left|1-c_{1}\left(1-x^{2}\right)\right| d x=(2 / 3)\left(4 s_{1}^{3}-3 s_{1}+1\right) /\left(1-s_{1}^{2}\right)
$$

with $s_{1}=2 \sin \pi / 18$, i.e., (1.1) can be replaced by the equality

$$
\min _{f \in(\mathfrak{L}-\mathfrak{P})_{1}} \int_{-1}^{1}|1-f(x)| d x=\frac{2}{3} \frac{4 s_{1}^{3}-3 s_{1}+1}{1-s_{1}^{2}}=0.61407 \cdots .
$$

We include the following result because the case $p=2$ of Theorem 1 plays a crucial role in its proof. So it may be seen as an application.

For any continuous function $f:[-1,1] \longmapsto \mathbb{C}$ and any $p \in(0, \infty)$, let $\|f\|_{p}:=$ $\left(2^{-1} \int_{-1}^{1}|f(x)|^{p} d x\right)^{1 / p}$; besides, let $\|f\|_{\infty}:=\max _{-1 \leq x \leq 1}|f(x)|$.

Theorem 2. If $f$ belongs to $\mathfrak{L}-\mathfrak{P}$ and has $x=-1$ and $x=1$ as consecutive zeros, then

$$
\|f\|_{1} \leq \sqrt{\frac{5}{6}}\|f\|_{2}
$$

where equality holds if and only if $f(z):=c\left(1-z^{2}\right), c \in \mathbb{R} \backslash\{0\}$.

Remark 2. Theorem 2 is an analogue of a result of Erdős and Grünwald [4]. They had proved that under the conditions of Proposition 1

$$
\|f\|_{1} \leq \frac{2}{3}\|f\|_{\infty}
$$

where equality holds if and only if $f(z):=c\left(1-z^{2}\right), c \in \mathbb{R} \backslash\{0\}$. 
Let $P_{0}, P_{1}, \ldots, P_{n}, \ldots$ be the orthonormal set of Legendre polynomials, i.e.,

$$
P_{n}(x):=\sqrt{\frac{2 n+1}{2}} \frac{1}{2^{n}} \sum_{\nu=0}^{\left[\frac{n}{2}\right]}(-1)^{\nu}\left(\begin{array}{c}
n \\
\nu
\end{array}\right)\left(\begin{array}{c}
2 n-2 \nu \\
n
\end{array}\right) x^{n-2 \nu} \quad(n=0,1,2, \ldots) .
$$

Consider an arbitrary function $f$ belonging to $\mathfrak{L}-\mathfrak{P}$ having $x=-1$ and $x=1$ as consecutive zeros and denote by $b_{0}, b_{1}, \ldots, b_{n}, \ldots$ its Legendre-Fourier coefficients, i.e.,

$$
b_{n}:=\int_{-1}^{1} f(x) P_{n}(x) d x, \quad n=0,1,2, \ldots
$$

Then [3, Theorem 8.9.1]

$$
\|f\|_{1}=\frac{1}{\sqrt{2}}\left|b_{0}\right|, \quad\|f\|_{2}=\sqrt{\frac{1}{2} \sum_{n=0}^{\infty}\left|b_{n}\right|^{2}}
$$

so

$$
\left|b_{0}\right|^{2} \leq 5 \sum_{n=1}^{\infty}\left|b_{n}\right|^{2}
$$

which says in particular that $\left|b_{0}\right|$ can be estimated from above, in terms of the coefficients $b_{1}, b_{2}, \ldots, b_{n}, \ldots$ Thus, we have:

Corollary 1. If $b_{0}, b_{1}, \ldots, b_{n}, \ldots$ are the Legendre-Fourier coefficients of a function $f$ belonging to $\mathfrak{L}-\mathfrak{P}$ having $x=-1$ and $x=1$ as consecutive zeros, then (2.3) holds. The inequality is sharp.

\section{Preparatory lemmas}

3.1. The class $\mathcal{P}_{\mathrm{R}^{+}, n}$ and the set $\mathfrak{M}_{n, p}$. Let $\mathcal{P}_{\mathrm{R}^{+}, n}$ consist of polynomials in $\mathcal{P}_{\mathrm{R}^{+}}$ whose degree does not exceed $n+2$ and consider the auxiliary problem of determining

$$
\mu_{n, p}:=\inf \left\{\int_{-1}^{1}|1-f(x)|^{p} d x: f \in \mathcal{P}_{\mathrm{R}^{+}, n}\right\}, \quad p>0 .
$$

Each $f$ in $\mathcal{P}_{\mathrm{R}^{+}, n}$ can be written in the form

$$
f(x):=c\left(1-x^{2}\right) \prod_{k=1}^{n}\left(1-t_{k} x\right), \quad c>0,-1 \leq t_{k} \leq 1 \text { for } 1 \leq k \leq n .
$$

It is clear that if $f(0)=c<2^{-n}$, then $\|f\|_{\infty}=M<1$. Consequently,

$$
0<1-(1+\epsilon) f(x)<1-f(x), \quad-1<x<1,
$$

for $0<\epsilon<M^{-1}-1$, so

$$
\mu_{n, p}=\inf \left\{\int_{-1}^{1}|1-f(x)|^{p} d x: f \in \mathcal{P}_{\mathrm{R}^{+}, n}, f(0) \geq 2^{-n}\right\} .
$$

For any $p>0$ and $f$ as in (3.2), let

$$
\omega_{p}(f)=\Omega_{p}\left(c ; t_{1}, \ldots, t_{n}\right):=\int_{-1}^{1}\left|1-c\left(1-x^{2}\right) \prod_{k=1}^{n}\left(1-t_{k} x\right)\right|^{p} d x
$$


The function $\omega_{p}(f):=\Omega_{p}(c ; \mathbf{t})$ where $\mathbf{t}:=\left(t_{1}, \ldots, t_{n}\right)$ is continuous on $[0, \infty) \times[-1,1]^{n}$. We note that

$$
\Omega_{p}(c ; \mathbf{t}) \geq\left\{\begin{array}{cc}
c^{p} \int_{-1}^{1}\left(\left(1-x^{2}\right)(1-|x|)^{n}\right)^{p} d x-2 & \text { if } 0<p<1 \\
\left(c\left(\int_{-1}^{1}\left(\left(1-x^{2}\right)(1-|x|)^{n}\right)^{p} d x\right)^{\frac{1}{p}}-2^{\frac{1}{p}}\right)^{p} & \text { if } 1 \leq p<\infty
\end{array}\right.
$$

so, given any $L<\infty$, we can find $\gamma_{L, n, p}>1$ such that $\Omega_{p}(c ; \mathbf{t})>L$ for all $c>\gamma_{L, n, p}$ and all $\mathbf{t}$ in $[-1,1]^{n}$. Hence, there exists $c_{p, n} \in(0, \infty)$ such that

$$
\mu_{n, p}=\inf \left\{\Omega_{p}(c ; \mathbf{t}): c \in\left[2^{-n}, c_{p, n}\right], \mathbf{t} \in[-1,1]^{n}\right\} .
$$

The set $E_{n}:=\left[2^{-n}, c_{p, n}\right] \times[-1,1]^{n}$ being compact, the infimum of $\Omega_{p}(c ; \mathbf{t})$ on $E_{n}$ is attained for some $c=c_{p, n}^{*} \in\left[2^{-n}, c_{p, n}\right]$ and some $\mathbf{t}=\mathbf{t}^{*} \in[-1,1]^{n}$. Hence, the infimum in (3.1) is attained for at least one polynomial in $\mathcal{P}_{\mathrm{R}^{+}, n}$. Let us denote by $\mathfrak{M}_{n, p}$ the set of all such polynomials and define

$$
\mathfrak{M}_{p}:=\cup_{n=1}^{\infty} \mathfrak{M}_{n, p}
$$

Further, for any $p>0$, let

$$
\mu_{p}:=\inf \left\{\int_{-1}^{1}|1-f(x)|^{p}: f \in \mathcal{P}_{\mathrm{R}^{+}}\right\} .
$$

Then it is clear that

$$
\mu_{p}:=\inf \left\{\int_{-1}^{1}|1-f(x)|^{p}: f \in \mathfrak{M}_{p}\right\} .
$$

3.2. An essential property of polynomials in $\mathfrak{M}_{p}$. From Rolle's theorem, it follows that an arbitrary $f$ in $\mathcal{P}_{\mathrm{R}^{+}}$has one and only one critical point in $(-1,1)$, so the graph of $y=f(x)$ on $(-1,1)$ cannot have more than two points of intersection with the line $y=1$. It is not self-evident but if $f \in \mathfrak{M}_{p}$, then there must be two such points $\left(\xi_{1}, 1\right),\left(\xi_{2}, 1\right)$ where $-1<\xi_{1}<\xi_{2}<1$. To see this, note that if $f(x):=c_{p}^{*}\left(1-x^{2}\right) g(x)$ belongs to $\mathfrak{M}_{p}$, then because $0<c_{p}^{*}<\infty$, the partial derivative of $\int_{-1}^{1}\left|1-c\left(1-x^{2}\right) g(x)\right|^{p} d x$ with respect to $c$ must vanish at $c_{p}^{*}$. But

$$
\begin{aligned}
\frac{\partial}{\partial c} \int_{-1}^{1}\left|1-c\left(1-x^{2}\right) g(x)\right|^{p} d x= & -p \int_{-1}^{1} \operatorname{sgn}\left(1-c\left(1-x^{2}\right) g(x)\right) \\
& \times\left|1-c\left(1-x^{2}\right) g(x)\right|^{p-1}\left(1-x^{2}\right) g(x) d x,
\end{aligned}
$$

so it cannot vanish for $c=c_{p}^{*}$ unless $1-c_{p}^{*}\left(1-x^{2}\right) g(x)$ changes sign in $(-1,1)$, and then it must do so twice. So in looking for the infimum of $\int_{-1}^{1}|1-f(x)|^{p} d x$ over $\mathcal{P}_{\mathrm{R}^{+}, n}$, we may restrict ourselves to polynomials whose graph on $(-1,1)$ intersects the line $y=1$ in two distinct points.

3.3. A reformulation of the problem. Given two points $\xi_{1}, \xi_{2}$ in $(-1,1)$, let $\mathcal{P}_{\mathrm{R}^{+}, n, \xi_{1}, \xi_{2}}$ consist of all those polynomials in $\mathcal{P}_{\mathrm{R}^{+}, n}$ whose graph on $(-1,1)$ intersects the line $y=1$ in the points $\left(\xi_{1}, 1\right)$ and $\left(\xi_{2}, 1\right)$. The set $\mathcal{P}_{\mathrm{R}^{+}, n, \xi_{1}, \xi_{2}}$ may be empty for small values of $n$. For example, $\mathcal{P}_{\mathrm{R}^{+}, 1, \xi_{1}, \xi_{2}}=\emptyset$ if $\xi_{1}=0,(\sqrt{5}-1) / 2 \leq \xi_{2}<1$. However, we claim that for each pair $\xi_{1}, \xi_{2}$ where $-1<\xi_{1}<\xi_{2}<1$, there exists a smallest positive integer $n^{*}=n^{*}\left(\xi_{1}, \xi_{2}\right)$ such that $\mathcal{P}_{\mathrm{R}^{+}, n, \xi_{1}, \xi_{2}} \neq \emptyset$ for each $n \geq n^{*}$. It 
suffices to show that for some $c>0$ and some $t \in(-1,1)$, depending on $\xi_{1}, \xi_{2}$, and $n$, the polynomial $f(x):=c\left(1-x^{2}\right)(1-t x)^{n}$ satisfies

$$
f\left(\xi_{1}\right)=f\left(\xi_{2}\right)=1
$$

for all large $n$. Let $n \geq 1$. For (3.6) to hold, we must have

$$
t=\frac{a-1}{a \xi_{2}-\xi_{1}}
$$

where $a=\left(\left(1-\xi_{2}^{2}\right) /\left(1-\xi_{1}^{2}\right)\right)^{1 / n}$. It is clear that $a \rightarrow 1$ as $n \rightarrow \infty$, so

$$
\left|(a-1) /\left(a \xi_{2}-\xi_{1}\right)\right| \leq 1
$$

for all large $n$. For each such $n,(3.6)$ holds for $f(x):=c\left(1-x^{2}\right)(1-t x)^{n}$ if and only if

$$
\begin{gathered}
t=t\left(n, \xi_{1}, \xi_{2}\right):=\frac{\left(1-\xi_{2}^{2}\right)^{1 / n}-\left(1-\xi_{1}^{2}\right)^{1 / n}}{\left(1-\xi_{2}^{2}\right)^{1 / n} \xi_{2}-\left(1-\xi_{1}^{2}\right)^{1 / n} \xi_{1}}, \\
c=c\left(n, \xi_{1}, \xi_{2}\right):=\frac{\left(\xi_{2}\left(1-\xi_{2}^{2}\right)^{1 / n}-\xi_{1}\left(1-\xi_{1}^{2}\right)^{1 / n}\right)^{n}}{\left(1-\xi_{1}^{2}\right)\left(1-\xi_{2}^{2}\right)\left(\xi_{2}-\xi_{1}\right)^{n}} .
\end{gathered}
$$

For each $n \geq n^{*}\left(\xi_{1}, \xi_{2}\right)$, let

$$
\Phi_{n, \xi_{1}, \xi_{2}}(x):=c\left(n, \xi_{1}, \xi_{2}\right)\left(1-x^{2}\right)\left(1-t\left(n, \xi_{1}, \xi_{2}\right) x\right)^{n}
$$

with $t\left(n, \xi_{1}, \xi_{2}\right), c\left(n, \xi_{1}, \xi_{2}\right)$ as in (3.7), (3.8), respectively. In particular, if $\xi_{2}=-\xi_{1}$, then $t\left(n, \xi_{1}, \xi_{2}\right)=0$ and $c\left(n, \xi_{1}, \xi_{2}\right)=1 /\left(1-\xi_{1}^{2}\right)$ for all $n \geq 0$.

Recalling the definition of $\mu_{p}$, we see that if

$$
\mu_{n, \xi_{1}, \xi_{2}, p}:=\inf \left\{\int_{-1}^{1}|1-f(x)|^{p} d x: f \in \mathcal{P}_{\mathrm{R}^{+}, n, \xi_{1}, \xi_{2}}\right\}, \quad p>0
$$

then

$$
\mu_{p}=\inf _{-1<\xi_{1}<\xi_{2} \leq 1} \inf _{n \geq n^{*}\left(\xi_{1}, \xi_{2}\right)} \mu_{n, \xi_{1}, \xi_{2}, p}
$$

3.4. Two crucial lemmas. Given $\xi_{1}$, $\xi_{2}$ such that $-1<\xi_{1}<\xi_{2}<1$, the infimum $\mu_{n, \xi_{1}, \xi_{2}, p}$ of $\int_{-1}^{1}|1-f(x)|^{p} d x$ over all polynomials $f \in \mathcal{P}_{\mathrm{R}^{+}, n, \xi_{1}, \xi_{2}}$ for any fixed $n \geq$ $n^{*}\left(\xi_{1}, \xi_{2}\right)$ is attained. To see this, observe that if $f \in \mathcal{P}_{\mathrm{R}^{+}, n, \xi_{1}, \xi_{2}}$, then for each $x \in(-1,1)$, we have

$$
f(x)=\frac{f(x)}{f\left(\xi_{1}\right)}<\frac{1-x^{2}}{1-\left|\xi_{1}\right|^{2}} \frac{2^{n}}{\left(1-\left|\xi_{1}\right|\right)^{n}} \leq \frac{2^{n}}{\left(1-\left|\xi_{1}\right|\right)^{n+1}\left(1+\left|\xi_{1}\right|\right)}
$$

In particular,

$$
f(0)<\frac{2^{n}}{\left(1-\left|\xi_{1}\right|\right)^{n+1}\left(1+\left|\xi_{1}\right|\right)} .
$$

It follows that if $f(z):=\sum_{\nu=0}^{n} a_{\nu} z^{\nu}$, then

$$
\left|a_{\nu}\right|<u\left(n, \nu, \xi_{1}\right):=\left(\begin{array}{l}
n \\
\nu
\end{array}\right) \frac{2^{n}}{\left(1-\left|\xi_{1}\right|\right)^{n+1}\left(1+\left|\xi_{1}\right|\right)}, \quad 0 \leq \nu \leq n .
$$


For each $j \in \mathbb{N}$, there exists a polynomial $h_{j}(z):=\sum_{\nu=0}^{n} a_{\nu, j} z^{\nu}$ belonging to $\mathcal{P}_{\mathrm{R}^{+}, n, \xi_{1}, \xi_{2}}$ such that

$$
\int_{-1}^{1}\left|1-h_{j}(x)\right|^{p} d x<\mu_{n, \xi_{1}, \xi_{2}, p}+\frac{1}{j} .
$$

Since $\left|a_{\nu, j}\right|<u\left(n, \nu, \xi_{1}\right)$ for all $j \in \mathbb{N}$ and $0 \leq \nu \leq n$, we can use a standard argument to select a subsequence $\left\{h_{j_{1}}, \ldots, h_{j_{k}}, \ldots\right\}$ of $h_{j}$ converging uniformly on any compact subset of $\mathbb{C}$ to a polynomial $F$ of degree at most $n$. Since $h_{j}\left(\xi_{1}\right)=1$ for each $j$, we note that $F$ cannot be identically zero.

Note that $\mathcal{P}_{\mathbf{R}^{+}, 1, \xi_{1}, \xi_{2}}$ is either empty or consists of only one element, namely $\Phi_{1, \xi_{1}, \xi_{2}}$.

Lemma 1. Let $n \geq 2$. If $f \in \mathcal{P}_{\mathrm{R}^{+}, n, \xi_{1}, \xi_{2}}$ and $f \neq \Phi_{n, \xi_{1}, \xi_{2}}$, then for all $p>0$, we have

$$
\int_{-1}^{1}|1-f(x)|^{p} d x>\int_{-1}^{1}\left|1-\Phi_{n, \xi_{1}, \xi_{2}}(x)\right|^{p} d x .
$$

Proof. Let $F(z):=\left(1-z^{2}\right) \varphi(z)$ be a polynomial in $\mathcal{P}_{\mathrm{R}^{+}, n, \xi_{1}, \xi_{2}}$ for which $\mu_{n, \xi_{1}, \xi_{2}, p}$ is attained. First we observe that $\varphi$ cannot have zeros in $(-\infty,-1]$ and $[1, \infty)$ at the same time. Suppose it does. Let $\lambda_{1}$ be the smallest zero of $F$ and $\lambda_{l}$ the largest. For all sufficiently small $\epsilon>0$, the polynomial

$$
F_{\epsilon, 1}(x):=F(x)-\epsilon \frac{F(x)}{\left(x-\lambda_{1}\right)\left(x-\lambda_{l}\right)}\left(x-\xi_{1}\right)\left(x-\xi_{2}\right)
$$

belongs to $\mathcal{P}_{\mathrm{R}^{+}, n, \xi_{1}, \xi_{2}}$ and $F(x)<F_{\epsilon, 1}(x)<1$ if $x \in\left(-1, \xi_{1}\right) \cup\left(\xi_{2}, 1\right)$, whereas $1<F_{\epsilon, 1}(x)<F(x)$ if $x \in\left(\xi_{1}, \xi_{2}\right)$. Hence,

$$
\int_{-1}^{1}\left|1-F_{\epsilon, 1}(x)\right|^{p} d x<\int_{-1}^{1}|1-F(x)|^{p} d x .
$$

Assume that $\varphi$ has no zeros in $(-\infty,-1]$. We claim that $\varphi$ cannot have two or more distinct zeros in $[1, \infty)$. Suppose it does. Let $\lambda_{l}$ be the largest zero and $\lambda_{k}$ the largest but one. It is geometrically evident that for all small $\epsilon>0$, the polynomial

$$
F_{\epsilon, 2}(x):=F(x)+\epsilon \frac{F(x)}{\left(x-\lambda_{k}\right)\left(x-\lambda_{l}\right)}\left(x-\xi_{1}\right)\left(x-\xi_{2}\right)
$$

belongs to $\mathcal{P}_{\mathrm{R}^{+}, n, \xi_{1}, \xi_{2}}$ and $F(x)<F_{\epsilon, 2}(x)<1$ if $x \in\left(-1, \xi_{1}\right) \cup\left(\xi_{2}, 1\right)$, whereas $1<F_{\epsilon, 2}(x)<F(x)$ if $x \in\left(\xi_{1}, \xi_{2}\right)$. Hence,

$$
\int_{-1}^{1}\left|1-F_{\epsilon, 2}(x)\right|^{p} d x<\int_{-1}^{1}|1-F(x)|^{p} d x .
$$

We have proved that $F(x)$ must be of the form $c\left(1-x^{2}\right)(1+\alpha x)^{m}$ where $n^{*}\left(\xi_{1}, \xi_{2}\right) \leq$ $m \leq n$. If $\xi_{2}=-\xi_{1}$, then clearly $\alpha=0$ and $F(x) \equiv \Phi_{n, \xi_{1},-\xi_{1}}(x)=\left(1-x^{2}\right) /\left(1-\xi_{1}^{2}\right)$. We claim that if $\xi_{2} \neq-\xi_{1}$, i.e., $\alpha \neq 0$, then $m$ cannot be less than $n$. Suppose it is. For sufficiently small $\epsilon>0$, the polynomial

$$
F_{\epsilon, 3}(x):=F(x)+\epsilon \frac{F(x)}{1+\alpha x}\left(x-\xi_{1}\right)\left(x-\xi_{2}\right)
$$

belongs to $\mathcal{P}_{\mathrm{R}^{+}, n, \xi_{1}, \xi_{2}}$ and

$$
\int_{-1}^{1}\left|1-F_{\epsilon, 3}(x)\right|^{p} d x<\int_{-1}^{1}|1-F(x)|^{p} d x,
$$


which is a contradiction. Hence, $F$ must be the polynomial $\Phi_{n, \xi_{1}, \xi_{2}}$. It follows that $\mu_{n, \xi_{1}, \xi_{2}, p}$ is attained by one and only one polynomial, namely $\Phi_{n, \xi_{1}, \xi_{2}}$.

From Lemma 1, it follows that

$$
\mu_{p}=\inf _{-1<\xi_{1}<\xi_{2}<1} \inf _{n \geq n^{*}\left(\xi_{1}, \xi_{2}\right)} \int_{-1}^{1}\left|1-\Phi_{n, \xi_{1}, \xi_{2}}(x)\right|^{p} d x .
$$

Lemma 2. Let $-1<\xi_{1}<\xi_{2}<1$ and $p>0$. Then

$$
\mu_{n+1, \xi_{1}, \xi_{2}, p} \leq \mu_{n, \xi_{1}, \xi_{2}, p}
$$

for all $n \geq n^{*}\left(\xi_{1}, \xi_{2}\right)$. In (3.13), equality holds only if $\xi_{2}=-\xi_{1}$.

Proof. The case $\xi_{2}=-\xi_{1}$ is trivial; so let $\xi_{2} \neq-\xi_{1}$. Refer to (3.9) and assume that $1 / t\left(n, \xi_{1}, \xi_{2}\right) \geq 1$. If $1 / t\left(n, \xi_{1}, \xi_{2}\right)>1$, then for all $\epsilon>0$, the polynomial

$$
g_{\epsilon}(x)=\Phi_{n, \xi_{1}, \xi_{2}}(x)+\epsilon\left(1-x^{2}\right)\left(1-t\left(n, \xi_{1}, \xi_{2}\right) x\right)^{n-1}\left(x-\xi_{1}\right)\left(x-\xi_{2}\right)
$$

has a zero of multiplicity $n-1$ at $1 / t\left(n, \xi_{1}, \xi_{2}\right)$, simple zeros at $-1,1$, one simple zero slightly larger than $1 / t\left(n, \xi_{1}, \xi_{2}\right)$, and another simple zero which is positive and large. In the case where $t\left(n, \xi_{1}, \xi_{2}\right)$ is equal to 1 , the zeros of $g_{\epsilon}$ at $1 / t\left(n, \xi_{1}, \xi_{2}\right)$ and 1 have to be replaced by a zero of multiplicity $n$ at 1 . Hence,

$$
\int_{-1}^{1}\left|1-g_{\epsilon}(x)\right|^{p} d x<\int_{-1}^{1}\left|1-\Phi_{n, \xi_{1}, \xi_{2}}(x)\right|^{p} d x
$$

Since $g_{\epsilon}$ belongs to $\mathcal{P}_{\mathrm{R}^{+}, n+1, \xi_{1}, \xi_{2}}$ and has two distinct positive zeros, we conclude that

$$
\begin{aligned}
\int_{-1}^{1}\left|1-\Phi_{n+1, \xi_{1}, \xi_{2}}(x)\right|^{p} d x & <\int_{-1}^{1}\left|1-g_{\epsilon}(x)\right|^{p} d x \\
& <\int_{-1}^{1}\left|1-\Phi_{n, \xi_{1}, \xi_{2}}(x)\right|^{p} d x .
\end{aligned}
$$

The case $1 / t\left(n, \xi_{1}, \xi_{2}\right) \leq-1$ can be handled similarly.

3.5. A "reduction" of the problem. From the expression for $c\left(n, \xi_{1}, \xi_{2}\right)$ given in (3.8), it is easily checked that

$$
\lim _{n \rightarrow \infty} c\left(n, \xi_{1}, \xi_{2}\right)=c\left(\xi_{1}, \xi_{2}\right):=\frac{\left(1-\xi_{2}^{2}\right)^{\frac{\xi_{2}}{\xi_{2}-\xi_{1}}}\left(1-\xi_{1}^{2}\right)^{-\frac{\xi_{1}}{\xi_{2}-\xi_{1}}}}{\left(1-\xi_{1}^{2}\right)\left(1-\xi_{2}^{2}\right)} .
$$

Further, referring to (3.7) for the definition of $t\left(n, \xi_{1}, \xi_{2}\right)$, we note that $n t\left(n, \xi_{1}, \xi_{2}\right)$ tends to a finite limit as $n \rightarrow \infty$. Indeed,

$$
\lim _{n \rightarrow \infty} n t\left(n, \xi_{1}, \xi_{2}\right)=\rho\left(\xi_{1}, \xi_{2}\right):=\ln \left(\frac{1-\xi_{2}^{2}}{1-\xi_{1}^{2}}\right)^{\frac{1}{\xi_{2}-\xi_{1}}} .
$$

It follows that for any fixed $x$ in $[-1,1]$,

$$
\Phi_{n, \xi_{1}, \xi_{2}}(x) \rightarrow c\left(\xi_{1}, \xi_{2}\right)\left(1-x^{2}\right) e^{-\rho\left(\xi_{1}, \xi_{2}\right) x} \quad \text { as } n \rightarrow \infty .
$$

Besides, from the proof of Lemma 2, it is clear that for all $n \geq n^{*}\left(\xi_{1}, \xi_{2}\right)$

$$
\Phi_{n, \xi_{1}, \xi_{2}}(x) \leq\left\{\begin{array}{lll}
1 & \text { if } & x \in\left[-1, \xi_{1}\right) \cup\left(\xi_{2}, 1\right], \\
\Phi_{n^{*}\left(\xi_{1}, \xi_{2}\right), \xi_{1}, \xi_{2}}(x) & \text { if } & x \in\left[\xi_{1}, \xi_{2}\right] .
\end{array}\right.
$$

Hence, the functions $\left|1-\Phi_{n, \xi_{1}, \xi_{2}}(x)\right|^{p}$ are uniformly bounded on $[-1,1]$. We may use Lebesgue's bounded convergence theorem to conclude that 


$$
\begin{aligned}
\inf _{n \geq n^{*}\left(\xi_{1}, \xi_{2}\right)} \int_{-1}^{1}\left|1-\Phi_{n, \xi_{1}, \xi_{2}}(x)\right|^{p} d x & =\lim _{n \rightarrow \infty} \int_{-1}^{1}\left|1-\Phi_{n, \xi_{1}, \xi_{2}}(x)\right|^{p} d x \\
& =\int_{-1}^{1}\left|1-c\left(\xi_{1}, \xi_{2}\right)\left(1-x^{2}\right) e^{-\rho\left(\xi_{1}, \xi_{2}\right) x}\right|^{p} d x
\end{aligned}
$$

Hence, from (3.12),

$$
\mu_{p}=\inf _{-1<\xi_{1}<\xi_{2}<1} \int_{-1}^{1}\left|1-c\left(\xi_{1}, \xi_{2}\right)\left(1-x^{2}\right) e^{-\rho\left(\xi_{1}, \xi_{2}\right) x}\right|^{p} d x
$$

It is easily checked that $c\left(\xi_{1}, \xi_{2}\right)$ can take any positive value as $\xi_{1}, \xi_{2}$ are allowed to vary such that $-1<\xi_{1}<\xi_{2}<1$; besides, $\rho\left(\xi_{1}, \xi_{2}\right)$ can take any real value. Consequently,

$$
\begin{aligned}
\mu_{p} & \geq \inf _{c \geq 0, \rho \in \mathbb{R}} \int_{-1}^{1}\left|1-c\left(1-x^{2}\right) e^{-\rho x}\right|^{p} d x \\
& =\inf _{c \geq 0, \rho \geq 0} \int_{-1}^{1}\left|1-c\left(1-x^{2}\right) e^{\rho x}\right|^{p} d x .
\end{aligned}
$$

The functions $c\left(1-x^{2}\right) e^{\rho x}$ do not belong to $\mathcal{P}_{\mathrm{R}}+$ except when $\rho=0$. So determining the infimum on the right-hand side of (3.15) can solve our problem at least in the polynomial case if it occurs for $\rho=0$ and for no other $\rho$. We shall show that this is indeed the case if $p \geq 1$.

\section{Proof of Theorem 1}

We shall first assume that $f$ is a polynomial and start with (3.15). In our argument, the case $p=1$ has to be distinguished from the case $p>1$.

4.1. The case $p=1$. For sake of clarity, we divide the proof into several steps.

Step 1. First let $\rho$ be any given nonnegative number and let us minimize $F(c, \rho):=$ $\int_{-1}^{1}\left|1-c\left(1-x^{2}\right) e^{\rho x}\right| d x$ as $c$ is allowed to vary in $[0, \infty)$. Clearly, $F(c, \rho)$ tends to $+\infty$ as $c$ tends to $+\infty$. Furthermore, $F(c, \rho)<F(0, \rho)$ for all small positive values of $\rho$. Hence, the infimum of $F(c, \rho)$ over $[0, \infty)$ is attained at some finite point of the open interval $(0, \infty)$. At such a point, we must have

$$
-\frac{\partial F}{\partial c}=\int_{-1}^{1} \operatorname{sgn}\left(1-c\left(1-x^{2}\right) e^{\rho x}\right)\left(1-x^{2}\right) e^{\rho x} d x=0 .
$$

This can be satisfied for some $c$ only if the function $1-c\left(1-x^{2}\right) e^{\rho x}$ changes sign in $(-1,1)$, at least once. Indeed, it must do so exactly twice since it has only one critical point in the interval. Let $1-c\left(1-x^{2}\right) e^{\rho x}$ change sign in $(-1,1)$; denote by $\xi_{1, c}, \xi_{2, c}$ the points where it does and let $\xi_{1, c}<\xi_{2, c}$. We claim that (4.1) is satisfied for only one value of $c$. To see this, divide the region bounded by the curve $y=\left(1-x^{2}\right) e^{\rho x}$ and the interval $[-1,1]$ into three parts by the lines $x=\xi_{1, c}, x=\xi_{2, c}$. Denote by $\mathfrak{A}_{1}(c)$ the area of the part lying to the left of $x=\xi_{1, c}$, by $\mathfrak{A}_{2}(c)$ the area of the one lying to the right of $x=\xi_{2, c}$, and by $\mathfrak{A}_{3}(c)$ the area of the part in the middle. Then (4.1) can be written as

$$
\mathfrak{A}(c):=\mathfrak{A}_{1}(c)+\mathfrak{A}_{2}(c)+\mathfrak{A}_{3}(c)=0 .
$$

If $1 / c$ is small, then $\mathfrak{A}_{1}(c)+\mathfrak{A}_{2}(c)$ is small and $\mathfrak{A}_{3}(c)$ is relatively large; but as $1 / c$ increases, $\mathfrak{A}_{1}(c)+\mathfrak{A}_{2}(c)$ increases while $\mathfrak{A}_{3}(c)$ decreases. So there is one and only one 
value of $c$ for which $\mathfrak{A}(c)=0$. Thus, for each $\rho \geq 0$, the equation (4.1) has a unique root which we denote by $c(\rho)$. Let $\xi_{1}(\rho):=\xi_{1, c(\rho)}, \xi_{2}(\rho):=\xi_{2, c(\rho)}$.

Step 2. For each given $\rho \geq 0$,

$$
\begin{aligned}
\min _{c \geq 0} & \int_{-1}^{1}\left|1-c\left(1-x^{2}\right) e^{\rho x}\right| d x=\int_{-1}^{1}\left|1-c(\rho)\left(1-x^{2}\right) e^{\rho x}\right| d x \\
& =\int_{-1}^{1} \operatorname{sgn}\left(\left(x-\xi_{1}(\rho)\right)\left(x-\xi_{2}(\rho)\right)\right)\left(1-c(\rho)\left(1-x^{2}\right) e^{\rho x}\right) d x \\
& =\int_{-1}^{1} \operatorname{sgn}\left(\left(x-\xi_{1}(\rho)\right)\left(x-\xi_{2}(\rho)\right)\right) d x
\end{aligned}
$$

since

$$
\int_{-1}^{1} \operatorname{sgn}\left(\left(x-\xi_{1}(\rho)\right)\left(x-\xi_{2}(\rho)\right)\right)\left(1-x^{2}\right) e^{\rho x} d x=0
$$

Hence,

$$
\min _{c \geq 0} \int_{-1}^{1}\left|1-c\left(1-x^{2}\right) e^{\rho x}\right| d x=2-2\left(\xi_{2}(\rho)-\xi_{1}(\rho)\right) .
$$

We show that $\xi_{2}(\rho)-\xi_{1}(\rho)$ is a decreasing function of $\rho$ for all $\rho>0$. For this we make use of (4.2) and the two relationships

$$
\begin{aligned}
& c(\rho)\left(1-\xi_{1}^{2}(\rho)\right) e^{\rho \xi_{1}(\rho)}=1 \\
& c(\rho)\left(1-\xi_{2}^{2}(\rho)\right) e^{\rho \xi_{2}(\rho)}=1 .
\end{aligned}
$$

We note that $\xi_{2}(\rho)>0$ for all $\rho \geq 0$ since otherwise we would have

$$
\begin{aligned}
0 & =\int_{-1}^{1} \operatorname{sgn}\left(\left(x-\xi_{1}(\rho)\right)\left(x-\xi_{2}(\rho)\right)\right)\left(1-x^{2}\right) e^{\rho x} d x \\
& =\int_{-1}^{0} \operatorname{sgn}\left(\left(x-\xi_{1}(\rho)\right)\left(x-\xi_{2}(\rho)\right)\right)\left(1-x^{2}\right) e^{\rho x} d x+\int_{0}^{1}\left(1-x^{2}\right) e^{\rho x} d x \\
& >-\int_{-1}^{0}\left(1-x^{2}\right) e^{\rho x} d x+\int_{0}^{1}\left(1-x^{2}\right) e^{\rho x} d x \\
& \geq 0
\end{aligned}
$$

Since for $\rho>0$, we have

$$
\frac{1-\xi_{1}^{2}(\rho)}{1-\xi_{2}^{2}(\rho)}=e^{\rho\left(\xi_{2}(\rho)-\xi_{1}(\rho)\right)}>1,
$$

it follows that $\xi_{2}(\rho)>\left|\xi_{1}(\rho)\right|$, and so

$$
\xi_{2}(\rho)+\xi_{1}(\rho)>0, \quad \rho>0 .
$$

Of course, $\xi_{2}(0)+\xi_{1}(0)=0$.

We extend the definition of $F(c, \rho)$ by setting $F(c, \rho):=\int_{-1}^{1}\left|1-c\left(1-x^{2}\right) e^{\rho x}\right| d x$ for all $\rho \in \mathbb{R}$. Then, clearly $F(c,-\rho)=F(c, \rho)$. The definitions of $c(\rho), \xi_{1}(\rho)$, and $\xi_{2}(\rho)$ also extend in an obvious way to negative values of $\rho$. Furthermore, $c(-\rho)=c(\rho)$, $\xi_{1}(-\rho)=-\xi_{2}(\rho)$ and $\xi_{2}(-\rho)=-\xi_{1}(\rho)$. In particular, $\xi_{2}(\rho)-\xi_{1}(\rho)$ is an even function of $\rho$. 
Step 3. We now prove that $\xi_{1}(\rho), \xi_{2}(\rho)$, and $c(\rho)$ are continuously differentiable functions of $\rho$. For this we write (4.1) in the equivalent form

$$
\begin{aligned}
& F_{1}\left(\xi_{1}, \xi_{2}, c, \rho\right):=\int_{-1}^{1} \operatorname{sgn}\left(\left(x-\xi_{1}\right)\left(x-\xi_{2}\right)\right)\left(1-x^{2}\right) e^{\rho x} d x=0, \\
& F_{2}\left(\xi_{1}, \xi_{2}, c, \rho\right):=\ln \left(c\left(1-\xi_{1}^{2}\right) e^{\rho \xi_{1}}\right)=0 \\
& F_{3}\left(\xi_{1}, \xi_{2}, c, \rho\right):=\ln \left(c\left(1-\xi_{2}^{2}\right) e^{\rho \xi_{2}}\right)=0
\end{aligned}
$$

and apply the implicit function theorem [5, Theorem 9.18]. It suffices to show that

$$
\frac{\partial\left(F_{1}, F_{2}, F_{3}\right)}{\partial\left(\xi_{1}, \xi_{2}, c\right)}=\left|\begin{array}{lll}
\frac{\partial F_{1}}{\partial \xi_{1}} & \frac{\partial F_{1}}{\partial \xi_{2}} & \frac{\partial F_{1}}{\partial c} \\
\frac{\partial F_{2}}{\partial \xi_{1}} & \frac{\partial F_{2}}{\partial \xi_{2}} & \frac{\partial F_{2}}{\partial c} \\
\frac{\partial F_{3}}{\partial \xi_{1}} & \frac{\partial F_{3}}{\partial \xi_{2}} & \frac{\partial F_{3}}{\partial c}
\end{array}\right| \neq 0
$$

The calculations are fairly simple, and we get

$$
\begin{gathered}
\frac{\partial F_{1}}{\partial \xi_{1}}=2\left(1-\xi_{1}^{2}\right) e^{\rho \xi_{1}}, \quad \frac{\partial F_{1}}{\partial \xi_{2}}=-2\left(1-\xi_{2}^{2}\right) e^{\rho \xi_{2}}, \quad \frac{\partial F_{1}}{\partial c}=0, \\
\frac{\partial F_{2}}{\partial \xi_{1}}=-\frac{2 \xi_{1}}{1-\xi_{1}^{2}}+\rho, \quad \frac{\partial F_{2}}{\partial \xi_{2}}=0, \quad \frac{\partial F_{2}}{\partial c}=\frac{1}{c} \\
\frac{\partial F_{3}}{\partial \xi_{1}}=0, \quad \frac{\partial F_{3}}{\partial \xi_{2}}=-\frac{2 \xi_{2}}{1-\xi_{2}^{2}}+\rho, \quad \frac{\partial F_{3}}{\partial c}=\frac{1}{c} .
\end{gathered}
$$

So

$$
\frac{\partial\left(F_{1}, F_{2}, F_{3}\right)}{\partial\left(\xi_{1}, \xi_{2}, c\right)}=\frac{4}{c^{2}}\left(\frac{\xi_{2}}{1-\xi_{2}^{2}}-\frac{\xi_{1}}{1-\xi_{1}^{2}}\right)
$$

which is positive since $\xi /\left(1-\xi^{2}\right)$ is an increasing function of $\xi$ in $(-1,1)$.

Step 4. Now we prove that

$$
\xi_{2}^{\prime}(\rho)-\xi_{1}^{\prime}(\rho)=-\frac{1}{2} c(\rho) \int_{-1}^{1} \operatorname{sgn}\left(\left(x-\xi_{1}(\rho)\right)\left(x-\xi_{2}(\rho)\right)\right) x^{3} e^{\rho x} d x .
$$

Write (4.2) in the form

$$
\int_{\xi_{2}(\rho)}^{1}\left(1-x^{2}\right) e^{\rho x} d x-\int_{\xi_{1}(\rho)}^{\xi_{2}(\rho)}\left(1-x^{2}\right) e^{\rho x} d x+\int_{-1}^{\xi_{1}(\rho)}\left(1-x^{2}\right) e^{\rho x} d x=0
$$

and differentiate with respect to $\rho$ to obtain

$$
\begin{aligned}
-2 \xi_{2}^{\prime}(\rho)\left(1-\xi_{2}^{2}(\rho)\right) e^{\rho \xi_{2}(\rho)}+ & 2 \xi_{1}^{\prime}(\rho)\left(1-\xi_{1}^{2}(\rho)\right) e^{\rho \xi_{1}(\rho)} \\
& +\left\{\int_{-1}^{\xi_{1}(\rho)}-\int_{\xi_{1}(\rho)}^{\xi_{2}(\rho)}+\int_{\xi_{2}(\rho)}^{1}\right\} x\left(1-x^{2}\right) e^{\rho x} d x=0,
\end{aligned}
$$

which gives

$$
\xi_{2}^{\prime}(\rho)-\xi_{1}^{\prime}(\rho)=\frac{1}{2} c(\rho) \int_{-1}^{1} \operatorname{sgn}\left(\left(x-\xi_{1}(\rho)\right)\left(x-\xi_{2}(\rho)\right)\right)\left(x-x^{3}\right) e^{\rho x} d x .
$$


However, if $\rho>0$, then from (4.8) it follows that

$$
\int_{-1}^{1} \operatorname{sgn}\left(\left(x-\xi_{1}(\rho)\right)\left(x-\xi_{2}(\rho)\right)\right) x e^{\rho x} d x=\left(1-\xi_{2}^{2}(\rho)\right) e^{\rho \xi_{2}(\rho)}-\left(1-\xi_{1}^{2}(\rho)\right) e^{\rho \xi_{1}(\rho)}
$$

where the expression on the right is zero by (4.4) and (4.5). This proves that the integral on the left is zero for $\rho>0$; but by continuity, it must be zero for $\rho=0$ also, i.e.,

$$
\int_{-1}^{1} \operatorname{sgn}\left(\left(x-\xi_{1}(\rho)\right)\left(x-\xi_{2}(\rho)\right)\right) x e^{\rho x} d x=0, \quad \rho \geq 0
$$

so (4.7) holds.

Step 5. Next we show that for $\rho>0$,

$$
\begin{aligned}
\left(\frac{4}{\rho}+\right. & \left.\xi_{2}(\rho)+\xi_{1}(\rho)\right) \int_{-1}^{1} \operatorname{sgn}\left(\left(x-\xi_{1}(\rho)\right)\left(x-\xi_{2}(\rho)\right)\right) x^{3} e^{\rho x} d x \\
= & \int_{-1}^{1} \operatorname{sgn}\left(\left(x-\xi_{1}(\rho)\right)\left(x-\xi_{2}(\rho)\right)\right)\left(x-\xi_{1}(\rho)\right)\left(x-\xi_{2}(\rho)\right)\left(1-x^{2}\right) e^{\rho x} d x \\
& +\frac{2}{\rho c(\rho)}\left(\xi_{2}^{2}(\rho)-\xi_{1}^{2}(\rho)\right) .
\end{aligned}
$$

Note that

$$
\begin{aligned}
\left(x-\xi_{1}(\rho)\right)\left(x-\xi_{2}(\rho)\right)\left(1-x^{2}\right)= & 1-x^{4}+\left(\xi_{1}(\rho)+\xi_{2}(\rho)\right) x^{3} \\
& \left.\left.-\left(1-\xi_{1}(\rho) \xi_{2}(\rho)\right)\left(1-x^{2}\right)-\left(\xi_{1}(\rho)\right)+\xi_{2}(\rho)\right)\right) x .
\end{aligned}
$$

Hence, using (4.2) and (4.10), we obtain

$$
\begin{gathered}
\int_{-1}^{1} \operatorname{sgn}\left(\left(x-\xi_{1}(\rho)\right)\left(x-\xi_{2}(\rho)\right)\right)\left(x-\xi_{1}(\rho)\right)\left(x-\xi_{2}(\rho)\right)\left(1-x^{2}\right) e^{\rho x} d x \\
=\int_{-1}^{1} \operatorname{sgn}\left(\left(x-\xi_{1}(\rho)\right)\left(x-\xi_{2}(\rho)\right)\right)\left(1-x^{4}\right) e^{\rho x} d x+\left(\xi_{1}(\rho)+\xi_{2}(\rho)\right) \\
\quad \times \int_{-1}^{1} \operatorname{sgn}\left(\left(x-\xi_{1}(\rho)\right)\left(x-\xi_{2}(\rho)\right)\right) x^{3} e^{\rho x} d x
\end{gathered}
$$

It is easily seen that the first integral on the right-hand side is equal to

$$
\begin{aligned}
& -\frac{2}{\rho}\left\{\left(1-\xi_{2}^{4}(\rho)\right) e^{\rho \xi_{2}(\rho)}-\left(1-\xi_{1}^{4}(\rho)\right) e^{\rho \xi_{1}(\rho)}\right\} \\
& +\frac{4}{\rho} \int_{-1}^{1} \operatorname{sgn}\left(\left(x-\xi_{1}(\rho)\right)\left(x-\xi_{2}(\rho)\right)\right) x^{3} e^{\rho x} d x .
\end{aligned}
$$

Using (4.4) and (4.5), we see that

$$
\left(1-\xi_{2}^{4}(\rho)\right) e^{\rho \xi_{2}(\rho)}-\left(1-\xi_{1}^{4}(\rho)\right) e^{\rho \xi_{1}(\rho)}=\frac{1}{c(\rho)}\left(\xi_{2}^{2}(\rho)-\xi_{1}^{2}(\rho)\right)
$$

and so (4.12) is equivalent to (4.11).

Step 6. Now refer to (4.11). The integral on the right-hand side is positive since the integrand is nothing but $\left|\left(x-\xi_{1}(\rho)\right)\left(x-\xi_{2}(\rho)\right)\right|\left(1-x^{2}\right) e^{\rho x}$. Because of (4.6), the 
other quantity on the right-hand side of (4.11) is also positive. For the same reason, $4 / \rho+\xi_{2}(\rho)+\xi_{1}(\rho)>0$ and so

$$
\int_{-1}^{1} \operatorname{sgn}\left(\left(x-\xi_{1}(\rho)\right)\left(x-\xi_{2}(\rho)\right)\right) x^{3} e^{\rho x} d x>0 .
$$

Now from (4.7), it follows that

$$
\frac{d\left(\xi_{2}(\rho)-\xi_{1}(\rho)\right)}{d \rho}<0 \text { for } \rho>0,
$$

i.e., $\xi_{2}(\rho)-\xi_{1}(\rho)$ steadily decreases as $\rho$ increases in the open interval $(0, \infty)$. This, in conjunction with (4.3), shows that

$$
\min _{\rho \geq 0} \min _{c \geq 0} \int_{-1}^{1}\left|1-c\left(1-x^{2}\right) e^{\rho x}\right| d x=\min _{c \geq 0} \int_{-1}^{1}\left|1-c\left(1-x^{2}\right)\right| d x .
$$

We use it in (3.15) to obtain the inequality

$$
\inf _{f \in \mathcal{P}_{\mathrm{R}^{+}}} \int_{-1}^{1}|1-f(x)| d x \geq \min _{c \geq 0} \int_{-1}^{1}\left|1-c\left(1-x^{2}\right)\right| d x .
$$

The inequality can be replaced by equality since the function $c\left(1-x^{2}\right)$ belongs to $\mathcal{P}_{\mathrm{R}^{+}}$. The argument used in Section 3.2 can be used to see that the minimum of $\int_{-1}^{1}\left|1-c\left(1-x^{2}\right)\right| d x$, when $c$ is allowed to vary in $[0, \infty)$, is attained for one and only one value of $c$. With this we have proved Theorem 1 for $f \in \mathcal{P}_{\mathrm{R}^{+}}$and $p=1$.

4.2. The case $p>1$. The proof is similar to the one for $p=1$ but differs in details. Take any $p>1$ and keep it fixed.

Step 1. Let $\rho$ be any given nonnegative number and minimize

$$
G(c, \rho):=\int_{-1}^{1}\left|1-c\left(1-x^{2}\right) e^{\rho x}\right|^{p} d x
$$

as $c$ is allowed to vary in $[0, \infty)$. The minimum is attained at some finite point in $(0, \infty)$. At such a point, we must have

$$
\frac{1}{p c^{p-1}} \frac{\partial G}{\partial c}=\int_{-1}^{1}-\left|\frac{1}{c}-\left(1-x^{2}\right) e^{\rho x}\right|^{p-1} \operatorname{sgn}\left(1-c\left(1-x^{2}\right) e^{\rho x}\right)\left(1-x^{2}\right) e^{\rho x} d x=0 .
$$

So $1-c\left(1-x^{2}\right) e^{\rho x}$ must change sign at exactly two points in $(-1,1)$. Let $\xi_{1, c}, \xi_{2, c}$ be as in Step 1 of the case $p=1$. Here again, we claim that (4.13) is satisfied for only one value of $c$. For sake of simplicity, let us use $\psi(c, x)$ to denote the integrand in (4.13). Let $\theta_{\rho}:=1 /\left(\max _{-1 \leq x \leq 1}\left(1-x^{2}\right) e^{\rho x}\right)$. Then, for $c>\theta_{\rho}$, we may write

$$
\psi(c, x):=-\left|\left(1-x^{2}\right) e^{\rho x}-\frac{1}{c}\right|^{p-1} \operatorname{sgn}\left(1-c\left(1-x^{2}\right) e^{\rho x}\right)\left(1-x^{2}\right) e^{\rho x} .
$$

Let $\theta_{\rho}<c<c+\delta$ and sketch the graphs of $\left(1-x^{2}\right) e^{\rho x},\left|\left(1-x^{2}\right) e^{\rho x}-1 / c\right|$, and $\left|\left(1-x^{2}\right) e^{\rho x}-1 /(c+\delta)\right|$ on $(-1,1)$. Looking at the points in

$$
\left(-1, \xi_{1, c+\delta}\right],\left(\xi_{1, c+\delta}, \xi_{1, c}\right],\left(\xi_{1, c}, \xi_{2, c}\right),\left(\xi_{2, c}, \xi_{2, c+\delta}\right),\left[\xi_{2, c+\delta}, 1\right)
$$


we see that $\psi(c, x)<\psi(c+\delta, x)$ for all $x \in(-1,1)$. The same conclusion is obviously true for $0<c<c+\delta \leq \theta(\rho)$ as well. Since $\psi(c, x)$ is a continuous function of $c$, it follows that

$$
\psi(c, x)<\psi(c+\delta, x) \text { if } 0<c<c+\delta \text { and }-1<x<1 .
$$

Hence, $\int_{-1}^{1} \psi(c, x) d x<\int_{-1}^{1} \psi(c+\delta, x) d x$ if $0<c<c+\delta$. Note, in addition, that $\int_{-1}^{1} \psi(c, x) d x<0$ for $0<c \leq \theta_{\rho}$. Finally, we observe that $\xi_{1, c} \rightarrow-1, \xi_{2, c} \rightarrow 1$ as $c \rightarrow \infty$ and $\int_{\xi_{1, c}}^{\xi_{2, c}} \psi(c, x) d x$, which is positive, becomes larger than $-\int_{-1}^{\xi_{1, c}} \psi(c, x) d x-$ $\int_{\xi_{2, c}}^{1} \psi(c, x) d x$, i.e., $\int_{-1}^{1} \psi(c, x) d x$ is positive for all large $c$. These facts about $\int_{-1}^{1} \psi(c, x) d x$ allow us to conclude that (4.13) is satisfied for only one value of $c$ which we denote by $c(p, \rho)$. Let $\xi_{1}(\rho):=\xi_{1, c(p, \rho)}, \xi_{2}(\rho):=\xi_{2, c(p, \rho)}$.

Remark 3. For later use, we remark that here again $\xi_{1}(0)+\xi_{2}(0)=0$ and $\xi_{1}(\rho)+$ $\xi_{2}(\rho)>0$ for $\rho>0$.

Our ultimate aim is to show that $G(c(p, \rho), \rho)$ is an increasing function of $\rho$ for $\rho>0$.

We extend the definition of $G(c, \rho)$ by setting

$$
G(c, \rho):=\int_{-1}^{1}\left|1-c\left(1-x^{2}\right) e^{\rho x}\right|^{p} d x
$$

for all $\rho \in \mathbb{R}$. The definitions of $c(p, \rho), \xi_{1}(\rho), \xi_{2}(\rho)$ also extend in an obvious way to negative values of $\rho$. Clearly enough, $c(-\rho)=c(\rho), \xi_{1}(-\rho)=-\xi_{2}(\rho)$, and $\xi_{2}(-\rho)=$ $-\xi_{1}(\rho)$.

Step 2. If $p>1$ is given, then $c(p, \rho), \xi_{1}(\rho), \xi_{2}(\rho)$ are continuously differentiable functions of $\rho$. To see this, write (4.13) in the equivalent form

$$
\begin{aligned}
G_{1}\left(\xi_{1}, \xi_{2}, c, \rho\right) & :=\int_{-1}^{1}\left|\frac{1}{c}-\left(1-x^{2}\right) e^{\rho x}\right|^{p-1} \operatorname{sgn}\left(\left(x-\xi_{1}\right)\left(x-\xi_{2}\right)\right)\left(1-x^{2}\right) e^{\rho x} d x=0, \\
G_{2}\left(\xi_{1}, \xi_{2}, c, \rho\right) & :=c\left(1-\xi_{1}^{2}\right) e^{\rho \xi_{1}}-1=0, \\
G_{3}\left(\xi_{1}, \xi_{2}, c, \rho\right) & :=c\left(1-\xi_{2}^{2}\right) e^{\rho \xi_{2}}-1=0 .
\end{aligned}
$$

After making some elementary calculations, we obtain

$$
\begin{aligned}
\frac{\partial\left(G_{1}, G_{2}, G_{3}\right)}{\partial\left(\xi_{1}, \xi_{2}, c\right)}=-(- & \left.\frac{2 \xi_{1}}{1-\xi_{1}^{2}}+\rho\right)\left(-\frac{2 \xi_{2}}{1-\xi_{2}^{2}}+\rho\right)(p-1) \\
& \times \int_{-1}^{1}\left|1-c\left(1-x^{2}\right) e^{\rho x}\right|^{p-2}\left(1-x^{2}\right)^{2} e^{2 \rho x} d x
\end{aligned}
$$

which is different from zero, since $-2 x /\left(1-x^{2}\right)+\rho$ vanishes in $(-1,1)$ only at the point where $\left(1-x^{2}\right) e^{\rho x}$ assumes its maximum and not at $\xi_{1}$ or $\xi_{2}$. Hence, by the implicit function theorem $c(p, \rho), \xi_{1}(\rho)$, and $\xi_{2}(\rho)$ are continuously differentiable functions of $\rho$.

In order to show that $G(c(p, \rho), \rho)$ is an increasing function of $\rho$ for $\rho>0$, we calculate its derivative with respect to $\rho$. It is equal to $\frac{\partial G}{\partial c} c^{\prime}(\rho)+\frac{\partial G}{\partial \rho}$; but $\frac{\partial G}{\partial c}$ is zero since $c(p, \rho)$ is the root of (4.13). Hence,

$$
\begin{aligned}
\frac{d}{d \rho} G(c(p, \rho), \rho)=\rho c & (p, \rho) \int_{-1}^{1}\left|1-c(p, \rho)\left(1-x^{2}\right) e^{\rho x}\right|^{p-1} \\
& \times \operatorname{sgn}\left(\left(x-\xi_{1}(\rho)\right)\left(x-\xi_{2}(\rho)\right)\right)\left(x^{3}-x\right) e^{\rho x} d x .
\end{aligned}
$$


Step 3. Now we show that

$$
\int_{-1}^{1}\left|1-c(p, \rho)\left(1-x^{2}\right) e^{\rho x}\right|^{p-1} \operatorname{sgn}\left(\left(x-\xi_{1}(\rho)\right)\left(x-\xi_{2}(\rho)\right)\right) x e^{\rho x} d x=0 .
$$

For $c=c(p, \rho), \xi_{1}=\xi_{1}(\rho), \xi_{2}=\xi_{2}(\rho)$, we have

$$
\begin{aligned}
0= & \int_{-1}^{1}\left|1-c\left(1-x^{2}\right) e^{\rho x}\right|^{p-1} \operatorname{sgn}\left(\left(x-\xi_{1}\right)\left(x-\xi_{2}\right)\right)\left(1-x^{2}\right) e^{\rho x} d x \\
= & \frac{2}{\rho} \int_{-1}^{1}\left|1-c\left(1-x^{2}\right) e^{\rho x}\right|^{p-1} \operatorname{sgn}\left(\left(x-\xi_{1}\right)\left(x-\xi_{2}\right)\right) x e^{\rho x} d x \\
& -\frac{1}{c \rho}\left(\int_{-1}^{\xi_{1}}+\int_{\xi_{2}}^{1}\right)\left(1-c\left(1-x^{2}\right) e^{\rho x}\right)^{p-1} d\left(1-c\left(1-x^{2}\right) e^{\rho x}\right) \\
& +\frac{1}{c \rho} \int_{\xi_{1}}^{\xi_{2}}\left(c\left(1-x^{2}\right) e^{\rho x}-1\right)^{p-1} d\left(c\left(1-x^{2}\right) e^{\rho x}-1\right)
\end{aligned}
$$

from which (4.14) follows because

$$
c(p, \rho)\left(1-\xi_{1}^{2}\right) e^{\rho \xi_{1}}=1, \quad c(p, \rho)\left(1-\xi_{2}^{2}\right) e^{\rho \xi_{2}}=1 .
$$

So

$$
\begin{aligned}
\frac{d}{d \rho} G(c(p, \rho), \rho)=\rho & c(p, \rho) \int_{-1}^{1}\left|1-c(p, \rho)\left(1-x^{2}\right) e^{\rho x}\right|^{p-1} \\
& \times \operatorname{sgn}\left(\left(x-\xi_{1}(\rho)\right)\left(x-\xi_{2}(\rho)\right)\right) x^{3} e^{\rho x} d x
\end{aligned}
$$

and it would suffice to show that if $\rho>0$, then

$$
\int_{-1}^{1}\left|1-c(p, \rho)\left(1-x^{2}\right) e^{\rho x}\right|^{p-1} \operatorname{sgn}\left(\left(x-\xi_{1}(\rho)\right)\left(x-\xi_{2}(\rho)\right)\right) x^{3} e^{\rho x} d x>0
$$

Step 4. For $c=c(p, \rho), \xi_{1}=\xi_{1}(\rho), \xi_{2}=\xi_{2}(\rho)$, we clearly have

$$
\begin{aligned}
0< & \int_{-1}^{1}\left|1-c\left(1-x^{2}\right) e^{\rho x}\right|^{p-1} \operatorname{sgn}\left(\left(x-\xi_{1}\right)\left(x-\xi_{2}\right)\right)\left(\left(x-\xi_{1}\right)\left(x-\xi_{2}\right)\right)\left(1-x^{2}\right) e^{\rho x} d x \\
= & \int_{-1}^{1}\left|1-c\left(1-x^{2}\right) e^{\rho x}\right|^{p-1} \operatorname{sgn}\left(\left(x-\xi_{1}\right)\left(x-\xi_{2}\right)\right)\left(1-x^{4}\right) e^{\rho x} d x \\
& \quad+\left(\xi_{1}+\xi_{2}\right) \int_{-1}^{1}\left|1-c\left(1-x^{2}\right) e^{\rho x}\right|^{p-1} \operatorname{sgn}\left(\left(x-\xi_{1}\right)\left(x-\xi_{2}\right)\right) x^{3} e^{\rho x} d x
\end{aligned}
$$

by (4.14) and (4.15). Writing $1-x^{4}$ in the form

$$
1-x^{2}+\frac{1}{\rho}\left(-2 x+\rho\left(1-x^{2}\right)+2 x\right) x^{2}
$$

and using (4.15), we see that (4.17) is equivalent to 


$$
\begin{aligned}
0< & \frac{1}{\rho} \int_{-1}^{1}\left|1-c\left(1-x^{2}\right) e^{\rho x}\right|^{p-1} \operatorname{sgn}\left(\left(x-\xi_{1}\right)\left(x-\xi_{2}\right)\right)\left(-2 x+\rho\left(1-x^{2}\right)\right) x^{2} e^{\rho x} d x \\
& +\left(\xi_{1}+\xi_{2}+\frac{2}{\rho}\right) \int_{-1}^{1}\left|1-c\left(1-x^{2}\right) e^{\rho x}\right|^{p-1} \operatorname{sgn}\left(\left(x-\xi_{1}\right)\left(x-\xi_{2}\right)\right) x^{3} e^{\rho x} d x \\
= & \frac{2}{c p \rho} \int_{-1}^{1}\left|1-c\left(1-x^{2}\right) e^{\rho x}\right|^{p} x d x \\
& +\left(\xi_{1}+\xi_{2}+\frac{2}{\rho}\right) \int_{-1}^{1}\left|1-c\left(1-x^{2}\right) e^{\rho x}\right|^{p-1} \operatorname{sgn}\left(\left(x-\xi_{1}\right)\left(x-\xi_{2}\right)\right) x^{3} e^{\rho x} d x
\end{aligned}
$$

But

$$
\begin{gathered}
\int_{-1}^{1}\left|1-c\left(1-x^{2}\right) e^{\rho x}\right|^{p} x d x=\int_{-1}^{1}\left|1-c\left(1-x^{2}\right) e^{\rho x}\right|^{p-1} \operatorname{sgn}\left(\left(x-\xi_{1}\right)\left(x-\xi_{2}\right)\right) \\
\times\left(1-c\left(1-x^{2}\right) e^{\rho x}\right) x d x
\end{gathered}
$$

So in view of (4.14), inequality (4.18) becomes

$$
\begin{aligned}
0< & \frac{2}{c p \rho} \int_{-1}^{1}\left|1-c\left(1-x^{2}\right) e^{\rho x}\right|^{p-1} \operatorname{sgn}\left(\left(x-\xi_{1}\right)\left(x-\xi_{2}\right)\right) x d x \\
& +\left(\xi_{1}+\xi_{2}+\frac{2}{\rho}+\frac{2}{p \rho}\right) \int_{-1}^{1}\left|1-c\left(1-x^{2}\right) e^{\rho x}\right|^{p-1} \operatorname{sgn}\left(\left(x-\xi_{1}\right)\left(x-\xi_{2}\right)\right) x^{3} e^{\rho x} d x .
\end{aligned}
$$

But, as pointed out in Remark $3, \xi_{1}(\rho)+\xi_{2}(\rho)>0$ for $\rho>0$, and so (4.16) would follow if we had

$$
\int_{-1}^{1}\left|1-c\left(1-x^{2}\right) e^{\rho x}\right|^{p-1} \operatorname{sgn}\left(\left(x-\xi_{1}\right)\left(x-\xi_{2}\right)\right) x d x \leq 0 \quad \text { for } \rho>0 .
$$

We are going to show that this is indeed true.

Step 5. First let $\xi_{1} \xi_{2} \leq 0$. Consider the function

$$
h_{\xi_{1}, \xi_{2}}(x):=\frac{1}{\xi_{1}-\xi_{2}}\left|\begin{array}{ccc}
1 & \xi_{1} & \xi_{1} e^{\rho \xi_{1}} \\
1 & \xi_{2} & \xi_{2} e^{\rho \xi_{2}} \\
1 & x & x e^{\rho x}
\end{array}\right|=x e^{\rho x}-\beta x-\alpha
$$

where

$$
\alpha:=-\xi_{1} \xi_{2} \frac{e^{\rho \xi_{2}}-e^{\rho \xi_{1}}}{\xi_{2}-\xi_{1}} \geq 0, \quad \beta:=\frac{\xi_{2} e^{\rho \xi_{2}}-\xi_{1} e^{\rho \xi_{1}}}{\xi_{2}-\xi_{1}}>0
$$

Clearly, $\xi_{1}, \xi_{2}$ are zeros of $h_{\xi_{1}, \xi_{2}}$. We note that they are simple. Indeed, if $h_{\xi_{1}, \xi_{2}}^{\prime}(u)=0$ for some real $u$, then $(\rho u+1) e^{\rho u}=\beta>0$; so $\rho u+1>0$ and $e^{\rho u}=\beta /(\rho u+1)$. Hence,

$$
h_{\xi_{1}, \xi_{2}}(u):=u e^{\rho u}-\beta u-\alpha=-\frac{\rho \beta u^{2}}{\beta u+1}-\alpha \neq 0 .
$$

In addition, we note that $h_{\xi_{1}, \xi_{2}}$ has no other zeros in $[-1,1]$. For this, it would suffice to show that $h_{\xi_{1}, \xi_{2}}^{\prime}$ has only one zero in $(-1,1)$. The second derivative $h_{\xi_{1}, \xi_{2}}^{\prime \prime}$ 
has only one zero, namely $-2 / \rho$, and so $h_{\xi_{1}, \xi_{2}}^{\prime}$ can have only two real zeros; only one of them can lie in $(-1,1)$ if $-2 / \rho \leq-1$. So let $-1<-2 / \rho$, i.e., $\rho>2$. We observe that $h_{\xi_{1}, \xi_{2}}^{\prime}$ decreases on $(-\infty,-2 / \rho)$, and at the point -1 , it takes on the value $(-\rho+1) e^{-\rho}-\beta<-e^{-\rho}-\beta<0$; so it has no zero in $[-1,-2 / \rho]$. On $(-2 / \rho, \infty)$, the derivative $h_{\xi_{1}, \xi_{2}}^{\prime}(x)$ increases as $x$ increases and therefore cannot have more than one zero in $(-2 / \rho, 1]$.

It is now clear that $h_{\xi_{1}, \xi_{2}}(x)$ is positive on $\left(-1, \xi_{1}\right) \cup\left(\xi_{2}, 1\right)$ and negative on $\left(\xi_{1}, \xi_{2}\right)$. Therefore, by (4.14)

$$
\begin{aligned}
0< & \int_{-1}^{1}\left|1-c\left(1-x^{2}\right) e^{\rho x}\right|^{p-1} \operatorname{sgn}\left(\left(x-\xi_{1}\right)\left(x-\xi_{2}\right)\right) h_{\xi_{1}, \xi_{2}}(x) d x \\
= & \xi_{1} \xi_{2}\left(e^{\rho \xi_{2}}-e^{\rho \xi_{1}}\right) \int_{-1}^{1}\left|1-c\left(1-x^{2}\right) e^{\rho x}\right|^{p-1} \operatorname{sgn}\left(\left(x-\xi_{1}\right)\left(x-\xi_{2}\right)\right) d x \\
& \quad-\left(\xi_{2} e^{\rho \xi_{2}}-\xi_{1} e^{\rho \xi_{1}}\right) \int_{-1}^{1}\left|1-c\left(1-x^{2}\right) e^{\rho x}\right|^{p-1} \operatorname{sgn}\left(\left(x-\xi_{1}\right)\left(x-\xi_{2}\right)\right) x d x
\end{aligned}
$$

Since $\operatorname{sgn}\left(\left(x-\xi_{1}\right)\left(x-\xi_{2}\right)\right)\left(1-c\left(1-x^{2}\right) e^{\rho x}\right)=\left|1-c\left(1-x^{2}\right) e^{\rho x}\right|$, it follows from (4.15) that

$$
\int_{-1}^{1}\left|1-c\left(1-x^{2}\right) e^{\rho x}\right|^{p-1} \operatorname{sgn}\left(\left(x-\xi_{1}\right)\left(x-\xi_{2}\right)\right) d x=\int_{-1}^{1}\left|1-c\left(1-x^{2}\right) e^{\rho x}\right|^{p} d x .
$$

We thus obtain

$$
\begin{gathered}
\left(\xi_{2} e^{\rho \xi_{2}}-\xi_{1} e^{\rho \xi_{1}}\right) \int_{-1}^{1}\left|1-c\left(1-x^{2}\right) e^{\rho x}\right|^{p-1} \operatorname{sgn}\left(\left(x-\xi_{1}\right)\left(x-\xi_{2}\right)\right) x d x \\
\quad<\xi_{1} \xi_{2}\left(e^{\rho \xi_{2}}-e^{\rho \xi_{1}}\right) \int_{-1}^{1}\left|1-c\left(1-x^{2}\right) e^{\rho x}\right|^{p} d x \leq 0,
\end{gathered}
$$

which proves (4.19) in the case $\xi_{1} \xi_{2} \leq 0$.

Now let us verify (4.19) when $\xi_{1} \xi_{2}>0$, i.e., when $0<\xi_{1}<\xi_{2}$. Since $\rho>0$, we have $\left|1-c\left(1-x^{2}\right) e^{\rho x}\right|^{p-1}<\left|1-c\left(1-x^{2}\right) e^{-\rho x}\right|^{p-1}$ for $0<x<1$, and so

$$
\begin{aligned}
\int_{-1}^{1} \mid & -\left.c\left(1-x^{2}\right) e^{\rho x}\right|^{p-1} \operatorname{sgn}\left(\left(x-\xi_{1}\right)\left(x-\xi_{2}\right)\right) x d x \\
& =\left(\int_{-1}^{0}+\int_{0}^{\xi_{1}}-\int_{\xi_{1}}^{\xi_{2}}+\int_{\xi_{2}}^{1}\right)\left|1-c\left(1-x^{2}\right) e^{\rho x}\right|^{p-1} x d x \\
& <-\int_{\xi_{1}}^{\xi_{2}}\left|1-c\left(1-x^{2}\right) e^{\rho x}\right|^{p-1} x d x-\int_{\xi_{1}}^{\xi_{2}}\left|1-c\left(1-x^{2}\right) e^{-\rho x}\right|^{p-1} x d x<0 .
\end{aligned}
$$

With this we have proved that if $f \in \mathcal{P}_{\mathrm{R}^{+}}$, then, for $p>1$,

$$
\int_{-1}^{1}|1-f(x)|^{p} d x \geq \min _{c \geq 0} \int_{-1}^{1}\left|1-c\left(1-x^{2}\right)\right|^{p} d x .
$$

It is trivial to show that this minimum is attained for a unique $c$. The proof of Theorem 1 for functions belonging to $\mathcal{P}_{\mathrm{R}^{+}}$is now complete. 
4.3. Transcendental functions in $(\mathfrak{L}-\mathfrak{P})_{1}$. It remains to prove that $(2.1)$ holds for an arbitrary $f \in(\mathfrak{L}-\mathfrak{P})_{1}$ which is different from $c_{p}\left(1-x^{2}\right)$. Let

$$
f(x):=c\left(1-x^{2}\right) g(x) \text { with } g(0)=1
$$

be an arbitrary function in $(\mathfrak{L}-\mathfrak{P})_{1}$ which is not a polynomial. The argument used in Section 3.2 can be used to show that such a function $f$ (with prescribed $g$ ) cannot minimize $\int_{-1}^{1}|1-f(x)|^{p} d x$ unless the graph of $y=f(x)$ on $(-1,1)$ cuts the line $y=1$ at least twice. In fact, it must do so exactly twice since each function in $(\mathfrak{L}-\mathfrak{P})_{1}$ is the local uniform limit of a sequence of polynomials in $\mathcal{P}_{\mathrm{R}^{+}}$. Denote these points by $\xi_{1}, \xi_{2}$ and let $\xi_{1}<\xi_{2}$.

Case (i). Let $\xi_{2} \neq-\xi_{1}$. Find a sequence $f_{1}, f_{2}, \ldots, f_{n}, \ldots$ of polynomials where $f_{n} \in \mathcal{P}_{\mathrm{R}^{+}, n}$ such that $f_{n}$ tends to $f$ locally uniformly. For all large $n$, the graph of $y=f_{n}(x)$ cuts the line $y=1$ in two points which we denote by $\xi_{n, 1}$ and $\xi_{n, 2}$ with $\xi_{n, 1}<\xi_{n, 2}$. Then, clearly $\xi_{n, 1} \rightarrow \xi_{1}, \xi_{n, 2} \rightarrow \xi_{2}$ as $n \rightarrow \infty$. Now using (3.11) and (3.14), we obtain

$$
\int_{-1}^{1}\left|1-f_{n}(x)\right|^{p} d x \geq \int_{-1}^{1}\left|1-c\left(\xi_{n, 1}, \xi_{n, 2}\right)\left(1-x^{2}\right) e^{-\rho\left(\xi_{n, 1}, \xi_{n, 2}\right) x}\right|^{p} d x
$$

so

$$
\begin{aligned}
\int_{-1}^{1}|1-f(x)|^{p} d x & =\lim _{n \rightarrow \infty} \int_{-1}^{1}\left|1-f_{n}(x)\right|^{p} d x \\
& \geq \lim _{n \rightarrow \infty} \int_{-1}^{1}\left|1-c\left(\xi_{n, 1}, \xi_{n, 2}\right)\left(1-x^{2}\right) e^{-\rho\left(\xi_{n, 1}, \xi_{n, 2}\right) x}\right|^{p} d x \\
& =\int_{-1}^{1}\left|1-c\left(\xi_{1}, \xi_{2}\right)\left(1-x^{2}\right) e^{-\rho\left(\xi_{1}, \xi_{2}\right) x}\right|^{p} d x \\
& >\int_{-1}^{1}\left|1-c_{p}\left(1-x^{2}\right)\right|^{p} d x
\end{aligned}
$$

since $\xi_{2} \neq-\xi_{1}$ (see the end of Section 3.5).

Case (ii). If $\xi_{2}=-\xi_{1}=\xi$, then $f(x) /\left(1-x^{2}\right)<1 /\left(1-\xi^{2}\right)$ for all $x$ in $(-1,-\xi) \cup$ $(\xi, 1)$ and $f(x) /\left(1-x^{2}\right)>1\left(1-\xi^{2}\right)$ for $-\xi<x<\xi$. Hence

$$
\int_{-1}^{1}|1-f(x)|^{p} d x>\int_{-1}^{1}\left|1-\frac{1}{1-\xi^{2}}\left(1-x^{2}\right)\right|^{p} d x
$$

\section{Proof of Theorem 2}

From the case $p=2$ of Theorem 1 , it follows that for all real $\lambda$, we have

$$
2-2 \lambda \int_{-1}^{1} f(x) d x+\lambda^{2} \int_{-1}^{1} f^{2}(x) d x=\int_{-1}^{1}(1-\lambda f(x))^{2} d x \geq \frac{1}{3}
$$

where the inequality is strict unless $\lambda f(x)=\frac{5}{4}\left(1-x^{2}\right)$. So

$$
2 \lambda \int_{-1}^{1} f(x) d x-\lambda^{2} \int_{-1}^{1} f^{2}(x) d x \leq \frac{5}{3}
$$

for all real $\lambda$. Choosing $\lambda=\left(\int_{-1}^{1} f(x) d x\right) /\left(\int_{-1}^{1} f^{2}(x) d x\right)$, we obtain (2.2). The case of equality is easily discussed. 


\section{References}

1. J. G. Clunie, Convergence of polynomials with restricted zeros, Lecture Notes in Mathematics 1511, Springer-Verlag, Berlin, 1992, pp.100-105.

2. J. G. Clunie and A. B. J. Kuijlaars, Approximation by polynomials with restricted zeros, J. Approx. Theory 79 (1994), 109-124.

3. P. J. Davis, Interpolation and Approximation, 2nd ed., Blaisdell Publishing Company, New York, 1965; reprinted by Dover Publications, Inc., New York, 1975.

4. P. Erdős and T. Grünwald, On polynomials with only real zeros, Ann. of Math. 40 (1939), 537-548.

5. W. Rudin, Principles of Mathematical Analysis, 2nd ed., McGraw-Hill, Inc., New York, 1964.

Department of Mathematics, University of Sofia, James Boucher 5, 1126 Sofia, Bulgaria E-mail: ddryan@bgearn.acad.bg

Département de Mathématiques et de Statistique, Université de Montréal, Montréal H3C 3J7, CANADA

E-mail: rahmanqi@ere.UMontreal.CA 